\title{
Pulsars identified from the NRAO VLA Sky Survey
}

\author{
J.L. $\operatorname{Han}^{1,2,3}$ and W.W. $\operatorname{Tian}^{1,3}$ \\ 1 Beijing Astronomical Observatory, Chinese Academy of Sciences (CAS), Beijing 100012, China \\ 2 Beijing Astronomy Center, CAS-PKU, Beijing 100871, China \\ e-mail: jhan@bac.pku.edu.cn \\ 3 Max-Planck-Institut für Radioastronomie, Auf dem Hügel 69, D-53121 Bonn, Germany
}

Received June 26, 1997; accepted March 1, 1999

\begin{abstract}
We identified 97 strong pulsars from the NRAO VLA Sky Survey (NVSS) at $1.4 \mathrm{GHz}$ north of Dec(J2000) $=-40^{\circ}$. The total flux density, linear polarization intensity and polarization angle (PA) of all pulsars are extracted from the NVSS catalog. The well-calibrated PA measurement of 5 pulsars can be used for absolute PA calibrations in other observations. Comparing the source positions with those in the pulsar catalog, we got the first measurement of the proper motion upper limit of PSR B0031-07, which is $\mu_{\alpha} \cos \delta=-102 \pm 74{\text { mas } \mathrm{yr}^{-1}}^{-1}$ and $\mu_{\delta}=-105 \pm 78$ mas $\mathrm{yr}^{-1}$.
\end{abstract}

Key words: pulsars: general — polarization — surveys

\section{Introduction}

Compared with other types of radio sources, pulsars are known to have strong polarization, even up to $100 \%$ if one observes them with high time resolutions. Pulsar polarization would be smeared somehow if they are observed as continuum point sources over a duration much longer than a pulsar period, mainly because of the fast swing of polarization angle across a pulse profile. However, we will show in this paper that is not so serious as generally believed.

Pulsars have high (birth) velocities, on average $450 \mathrm{~km} \mathrm{~s}^{-1}$ (Lyne \& Lorimer 1994) and maybe up to $1600 \mathrm{~km} \mathrm{~s}^{-1}$ for individuals (e.g. Cordes \& Chernoff 1998), much faster than that of other types of stars (typically a few tens $\mathrm{km} \mathrm{s}^{-1}$ ). The high velocity was probably caused by the asymmetric kick during supernova explosion when a pulsar was born. This leads to a large proper motion for (nearby) pulsars. However, measuring the proper motion is not an easy task since the precise positions of a pulsar at well-separated epochs have to be measured.

Send offprint requests to: J.L. Han
Up to now, there are 96 pulsars with proper motion measurements (e.g. Taylor et al. 1993; Fomalont et al. 1997).

Recently, the National Radio Astronomical Observatory (NRAO) Very Large Array (VLA) Sky Survey (NVSS) has been finished, which covers the sky north of $\operatorname{Dec}(\mathrm{J} 2000)=-40^{\circ}$ at $1.4 \mathrm{GHz}$ (Condon et al. 1998). The survey detected more than 1.8 million sources, with polarization measurements, down to a flux density limit about $2.5 \mathrm{mJy}$. Observations have a resolution of $45^{\prime \prime}$, but the positional accuracy is a few arcsec for weak sources, and much better for strong sources. The observations were made with two IF channels at 1.365 and $1.435 \mathrm{GHz}$ with an effective bandwidth of $42 \mathrm{MHz}$ each. Most sources in the NVSS were observed in three pointings of $23 \mathrm{~s}$ each. The final sky map is the weighted sum from these pointings (Condon et al. 1998).

We had tried to identify the pulsars from the NVSS catalog, and then to investigate the pulsar polarization properties and proper motions from continuum observations. In the sky region covered by the NVSS, there are 520 known pulsars according to the updated pulsar catalog of Taylor et al. (1993). Updated catalog was kindly provided by Manchester. Using the latest version of the NVSS catalog (with 1814748 entries), we identified 97 strong pulsars according to positional coincidence. During revising this paper for publication, we noticed that similar identification work has been done by Kaplan et al. (1998), but they emphasized the other aspects, such as position accuracy, scintillation effects and completeness of detections. Comparing to Kaplan et al. (1998), we got 24 further new identifications. In the following, we will not repeat their work, but present our results in Sect. 2. We discuss briefly in Sect. 3 about scintillations (Sect. 3.1), pulsar polarization properties (Sect. 3.2), and proper motions (Sect. 3.3). We compared the pulsar positions with those from the pulsar catalog if the epochs were separated over more than 5 years, and got the upper limits of proper motion of 18 
pulsars, including one pulsar which has had no proper motion measurements previously.

\section{Identification and results}

We took positions of pulsars from the updated catalog of Taylor et al. (1993). PSR names in J2000, and B1950 if applicable, are given in the Cols. (1) and (2) of the Table 1. Their positions are given in Cols. (3) and (4), generally with an accuracy better than $0.1^{\prime \prime}$, but occasionally up to a few arcsec. These positions were determined by timing observations or interferimetric measurements at epoch for the position ${ }^{1}$ in Col. (5). For comparison, we list in Col. (6) the flux density at $1.4 \mathrm{GHz}$ from pulsar catalog, which were normally obtained from the average of several pulsar observation sessions to overcome scintillation effects. We searched for radio sources in the NVSS catalog within $30^{\prime \prime}$ angular distance around each of the 520 pulsar positions. Only 106 radio sources were found to match the positions and are probably pulsars. The positions of the NVSS sources are listed in Cols. (7) and (8). The angular offset from pulsar positions " $\Delta$ " in arcsec is given in Col. (9). The flux density and polarization parameters of the NVSS sources extracted from the NVSS catalog are listed in Cols. (10)-(13). A blank in these columns indicates no significant detection above the sensitivity limit of linear polarization of the NVSS $(\sim 0.5 \mathrm{mJy})$. We marked in Col. (14) if there was any further consideration during identification.

Note that the epochs for pulsar position in the pulsar catalog differ from that of NVSS observations. However, even if a pulsar has the largest proper motion, e.g. 400 mas per year, then after 20 years, the position offset would be $8^{\prime \prime}$. So, our search in $30^{\prime \prime}$ should not miss any known pulsar if it is detectable by the $\mathrm{NVSS}^{2}$.

On the other hand, the NVSS was done over a long period, from $\sim 1993$ to $\sim 1996$. We will take an approximate epoch MJD 49718 ( 1995.0) in following discussion. There should be only a very small position offset $\left(<1^{\prime \prime}\right)$ caused by pulsar proper motions, if any, over the NVSS observation period, much smaller than the position uncertainties of the NVSS sources listed in Table 1. If the position of a pulsar was measured at an epoch later than MJD 47000, we will not consider its proper motion during the identification process for the same reason.

\footnotetext{
1 There are two epochs in the pulsar catalog, one ("pepoch") for pulsar period and period derivatives and the other ("epoch") for pulsar position. If "epoch" was not available, we used the "pepoch" as instructed by Manchester (private communications).

${ }^{2}$ We missed 4 pulsars which appear in Table 1 of Kaplan et al. (1998): PSRs B1823-11, B1900-06, B1901+10, and B2323+63. Their position offsets to the NVSS sources or position uncertainties are too large $\left(>30^{\prime \prime}\right)$ to make significant assessment. For the same reason, we removed J1848+0651 from our sample which was included by Kaplan et al. (1998).
}

The first step for identification is to check the position offset $\Delta$. At this stage, we ignored the proper motion. If $\Delta$ is smaller than twice of the total position uncertainty, i.e. $\Delta \lesssim 2 \sqrt{\sigma_{\text {nvss }}^{2}+\sigma_{\text {psrcat }}^{2}}$, then we attribute the NVSS source as being a positive identification of a pulsar. This process yielded the first 90 positive detections. If any pulsar position was obtained at an epoch several years ago, the pulsar must have had only a very small proper motion so that the position offsets are not significant.

Now we consider the remaining 16 sources more carefully, which are marked with "?" in Col. (14) of Table 1.

Nine Confusion cases: (a) PSR B0531+21 (Crab) and PSR B1951+32 are confused by their associated supernova remnants. We marked them in Notes, i.e. Col. (14), of Table 1 with "SNR". (b) PSRs B1112+50, B1829-10 and B1831-00 are confused by their nearby strong sources which have much larger flux density (more than 10 times) than that from the pulsar catalog. One NVSS source was detected 28.1" (formally 7.8 $\sigma$ ) away from PSR B1920+21, too large to be proper motion for this distant pulsar (distance $\sim 12.5 \mathrm{kpc}$ ). We consider these detections unlikely and mark with "no" in Notes to stand for "no detection". (c) PSRs B1744-24A and J2129+1210A, (maybe also B1745-20 as indicated by Kaplan et al. 1998), are confused by other continuum sources in the host globular clusters Terzan-5 and M 15, (and NGC 6440?), respectively. They are marked with "glbc". (d) PSR B1718-35 is a marginal case, maybe confused by a source 19 ". 4 away, with $4.7 \sigma$ for position offset and $27.7 \mathrm{mJy}$ in flux (pulsar: $10.0 \mathrm{mJy}$ ).

Seven detection cases: (a) The position offset of the NVSS source to PSR B1831-04 is only $4.75^{\prime \prime}$ (formally $2.3 \sigma$, or $2.6 \sigma$ rather than $14 \sigma$ using the new position in Kaplan et al. 1998), much smaller than the beam size of the NVSS. Although Kaplan et al. (1998) suggested otherwise, we believe the pulsar is detected. The consistent flux densities of the pulsar and the NVSS source confirm the identification. We mark such a case as "yes" in the Notes. (b) PSRs B0823+26, B1133+16, B2016+28, (and B2154+40) have small position offsets caused by proper motions (see Sect. 3.3). (c) PSR B1820-31 is detected with a position offset of $12.5^{\prime \prime}=2.6 \sigma$, as confirmed by consistent flux density, and more importantly, by the highly linear polarization of the NVSS source. (d) A marginal case is the strong pulsar PSR B2020+28. The NVSS survey detected a very weak source $2.2 \sigma$ away, too weak to believe the identification (see more discussion below). However, highly linear polarization of the source suggests that it is the pulsar. We mark in the Notes "yes?" for this case.

In all, the NVSS detected 97 pulsars, including the 73 which appeared in Kaplan et al. (1998) and 24 new identifications ${ }^{3}$ marked with "** in the Notes.

\footnotetext{
${ }^{3}$ The PSR J1615-39 in Table 1 of Kaplan et al. (1998) is missing from the pulsar catalog available to us.
} 
Table 1. NVSS sources around pulsar positions

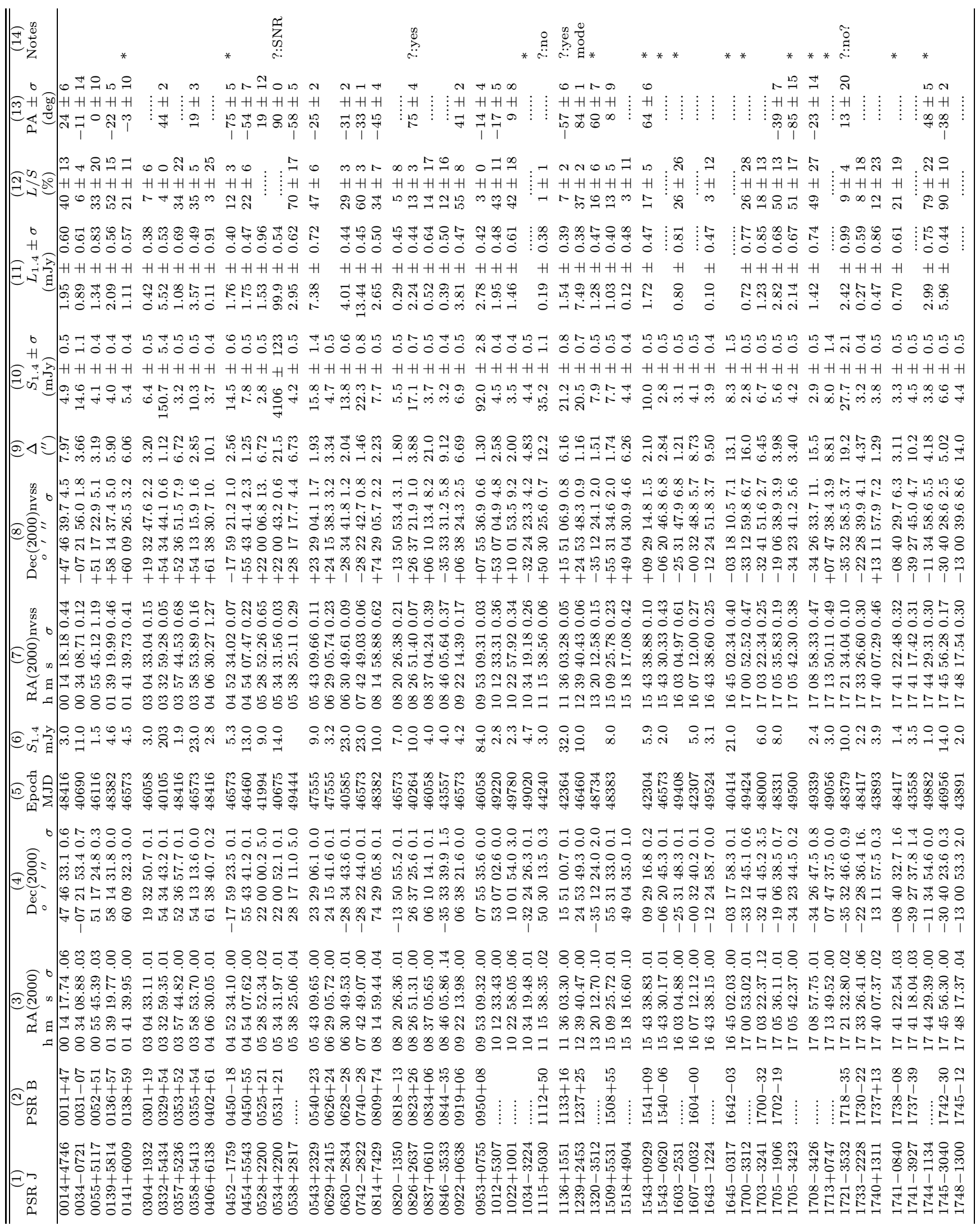


Table 1. continued

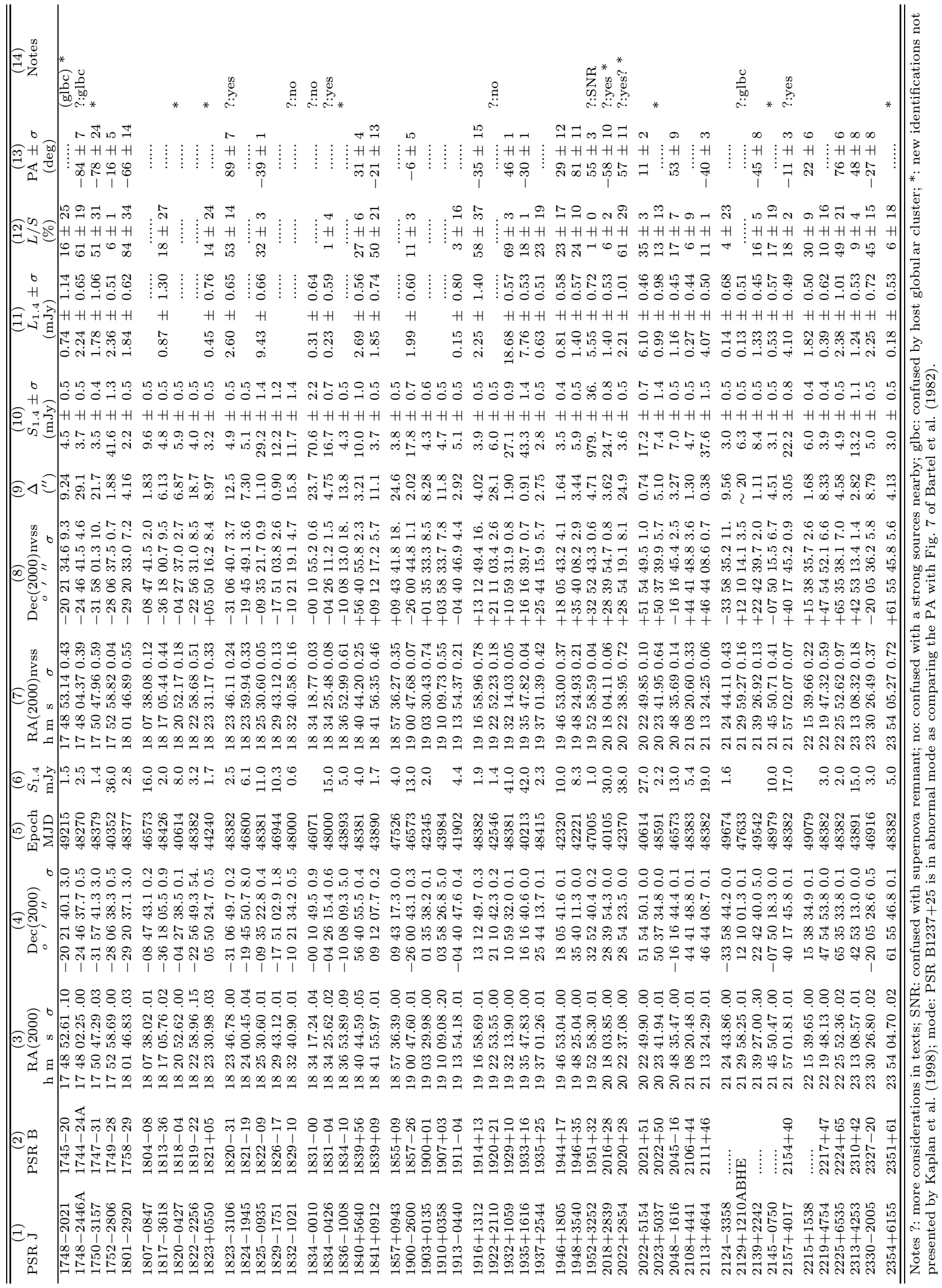




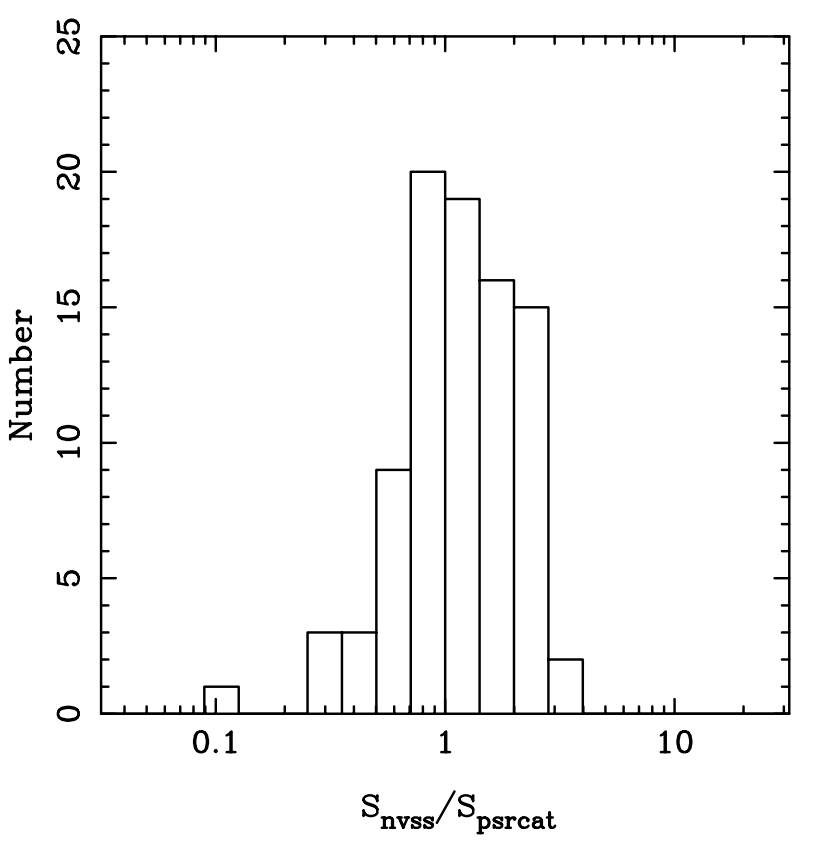

Fig. 1. The histograph for the flux comparison. Some pulsars (e.g. these in Table 1) have been missed in the left-half of the distribution (i.e. when $S_{\text {nvss }} / S_{\text {psrcat }}<1$ ), because scintillation makes them weaker than the NVSS sensitivity

\section{Discussion}

\subsection{Scintillation and undetected pulsars}

The VLA measurements of the flux densities $S_{1.4}$ of most identified pulsars, averaged over about $84 \mathrm{MHz}$ bandwidth and $3 \times 23 \mathrm{~s}$ in time, are comparable to the flux densities published in Lorimer et al. (1995) and Gould \& Lyne (1998). They are generally within a factor of 2 of the published densities (see Fig. 1), but sometimes up to a factor of 3 or more. Most of undetected pulsars $(\sim 400)$ have flux densities below 2 or $3 \mathrm{mJy}$. Interstellar scintillation (e.g. Gupta et al. 1994) both helps and hinders the detections (Cordes \& Lazio 1991). Some pulsars which have a flux density less than $2 \mathrm{mJy}$ in the pulsar catalog have been detected in the NVSS with a larger flux density. The scintillation effect is more obvious for strong pulsars. For example, PSR B2020+28 should be as strong as $38.0 \mathrm{mJy}$, but in the NVSS it appears to be a highly polarized source of $3.6 \pm 0.5 \mathrm{mJy}$. Among 61 pulsars with known flux densities larger than $5 \mathrm{mJy}$, about one fourth were missed by the NVSS (as listed in Table 2), some due to scintillation, some due to confusion (Condon, private communication).

\subsection{Polarization}

When pulsars are observed as continuum radio sources, the polarized intensity, $L$, and polarization position angle, PA, are calculated from the integrated $Q$ and $U$ values of
Table 2. Pulsars stronger than 5 mJy but not detected by the NVSS

\begin{tabular}{|c|c|c|c|c|}
\hline \multirow[t]{2}{*}{ PSR B } & $\overline{\mathrm{RA}(2000)}$ & $\operatorname{Dec}(2000)$ & $S_{1.4}$ & Notes \\
\hline & $\mathrm{h} \mathrm{m} \mathrm{s}$ & & $\mathrm{mJy}$ & \\
\hline $1937+21$ & 193938.55 & +213459.1 & 16.0 & Conf. \\
\hline $1800-21$ & 180351.35 & $\begin{array}{lll}-21 & 37 & 07.2\end{array}$ & 14.6 & Scin. \\
\hline $2319+60$ & 232155.19 & +602430.6 & 12 & Scin. \\
\hline $1845-01$ & 184824.00 & -012358.2 & 10 & Scin. \\
\hline $2255+58$ & 225757.70 & +590914.9 & 9.2 & Scin. \\
\hline $1839-04$ & $1842 \quad 26.49$ & -035959.2 & 8.5 & Conf. \\
\hline $1952+29$ & 195422.58 & +292317.9 & 8 & Scin. \\
\hline $1815-14$ & 181823.79 & -142235.9 & 7.4 & Scin. \\
\hline $1754-24$ & 175741.02 & -242156.8 & 7.1 & Conf. \\
\hline $2011+38$ & 201310.49 & +384544.8 & 6.4 & Scin. \\
\hline $1737-30$ & 174033.73 & -301541.9 & 6 & Scin. \\
\hline $1919+21$ & 192144.80 & +215301.8 & 6 & Scin. \\
\hline $1758-23$ & 180119.86 & -230616.8 & 5.7 & Scin. \\
\hline $1849+00$ & 185228.00 & +003155.9 & 5.2 & Scin. \\
\hline
\end{tabular}

the final images, i.e., over all the observation time and the bandwidth, so that

$L_{\mathrm{nvss}}=\sqrt{\left(\int_{t} Q\right)^{2}+\left(\int_{t} U\right)^{2}}$

and

$\mathrm{PA}_{\mathrm{nvss}}=\frac{1}{2} \frac{180}{\pi} \arctan \left(\frac{\int_{t} U}{\int_{t} Q}\right)$.

In pulsar observations, however, the total linearly polarized intensity is

$L_{\mathrm{psr}} \equiv \int_{t} \sqrt{Q^{2}+U^{2}}$

and the polarization position angle PA is

$\mathrm{PA}_{\mathrm{psr}}=\frac{1}{2} \frac{180}{\pi} \arctan \left(\frac{U}{Q}\right)$

for each pulse longitude. The PA often swings more than $90^{\circ}$ over a pulse. Since a positive value of $Q$ or $U$ in one part of a pulse may cancel a negative value in another part, it is believed that the pulsar emission is depolarized in contiuum observations. Furthermore, the bandwidth depolarization occurs for pulsars with high rotation measures. Therefore the $L / S$ in Table 1 should be taken as the lower limit of pulsar polarization.

Even so, pulsars are still the sources with the highest polarization compared to other kinds of objects (see Fig. 2). As seen from Table 1, some pulsars have very high linear polarization, such as PSRs B1742-30 ( $L / S \sim 90 \%)$ and PSR B1929+10 $(L / S \sim 63 \%)$, even after the smearing and depolarization.

Since the NVSS has very accurate absolute position angle calibrations $\left(<0.2^{\circ}\right)$, the well measured PA of a few pulsars (with error $\lesssim 2^{\circ}$ ) may help to make an absolute PA calibration in pulsar observations. One example 

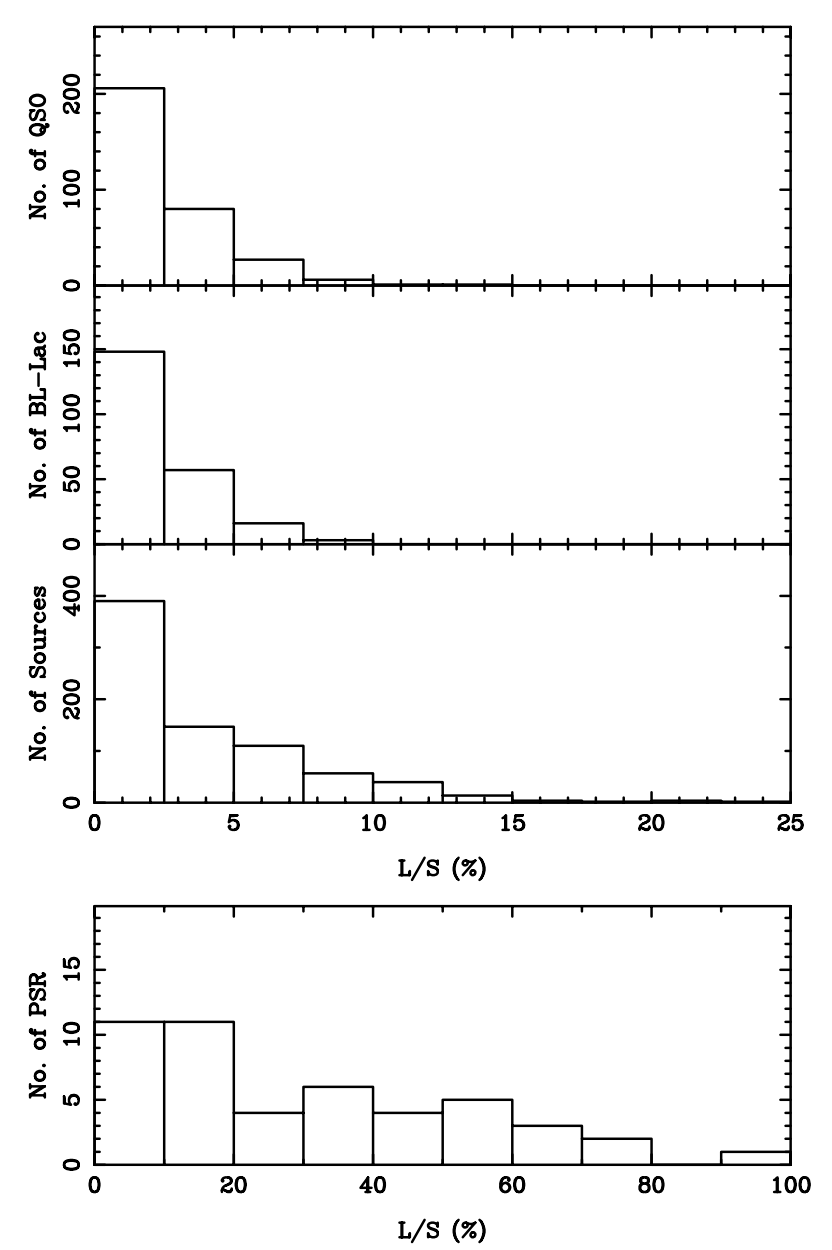

Fig. 2. Histograms of polarization percentage of a few kinds of objects: quasars, BL-Lac objects, all radio sources in one sky area, and pulsars. Note that the abscissa is up to $100 \%$ for pulsars, but just $25 \%$ for other objects

Table 3. Pulsar calibrators for absolute polarization angle

\begin{tabular}{|c|c|c|c|}
\hline PSR J & PSR B & $\begin{array}{c}\mathrm{PA}_{1400 \mathrm{MHz}}^{\mathrm{NVSS}} \\
\left(^{\circ}\right)\end{array}$ & $\begin{array}{c}R M \\
\left(\operatorname{rad~m^{-2}}\right)\end{array}$ \\
\hline $1932+1059$ & $1929+10$ & $46 \pm 1$ & $-6.1 \pm 1.0$ \\
\hline $0742-2822$ & $0740-28$ & $-33 \pm 1$ & $150.4 \pm 0.1$ \\
\hline $2022+5154$ & $2021+51$ & $11 \pm 2$ & $-6.5 \pm 0.9$ \\
\hline $0543+2329$ & $0540+23$ & $-25 \pm 2$ & $8.7 \pm 0.7$ \\
\hline $0630-2834$ & $0628-28$ & $-31 \pm 2$ & $46.2 \pm 0.1$ \\
\hline
\end{tabular}

is shown in Fig. 3. First, using the VLA measurements of $\mathrm{PA}$ at $1400 \mathrm{MHz}$ and the RM values, we calculated the averaged PA over the pulse at the observation frequency accordingly. Second, from the pulsar observations, we got PA for calibration pulsars using Eq. (2) from the pulse profiles (including interpulse if applicable) of Stokes parameters $Q$ and $U$. Third we compared them to get an offset which represents the instrument PA offset, and used it to calibrate all pulsar observations.

In Table 3, we listed 5 pulsars which can be used for calibration purposes. All of them have strong linear polar-

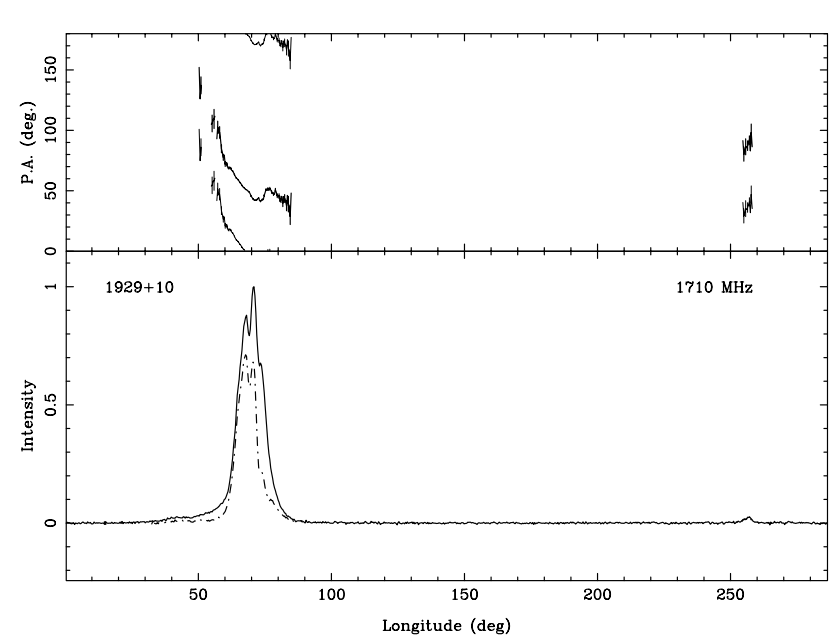

Fig. 3. Calibration for absolute polarization angle. Pulsar data were observed by von Hoensbroech \& Xilouris (1997). In the lower panel, the total intensity, $I$, and linearly polarized intensity, $L$, are plotted with a thick continuum line and a dot-dash line, respectively. (The interpulse is almost $100 \%$ polarized.) In the top panel, the original PA data are plotted with a thin line (and with an error bar on every second point), and the calibrated PA data are plotted with a thick line

ized intensity that can be easily detected, and their rotation measures RM are either quite small $\left(\lesssim 10 \mathrm{rad} \mathrm{m}^{-2}\right)$ or accurately measured $\left(\sigma_{\mathrm{RM}} \lesssim 1 \mathrm{rad} \mathrm{\textrm {m } ^ { - 2 }}\right)$. None of them has any mode-changing (e.g. PSR B1237+25 and PSR B1822+09) or complicated variations in PA across the profile (e.g. PSR B1933+16). All pulsars in Table 3 satisfy $\sigma_{\mathrm{PA}}+\sigma_{\mathrm{RM}} \cdot \delta\left(\lambda^{2}\right)<3^{\circ}$, where $\delta\left(\lambda^{2}\right)$ was the difference of the wavelengths squared, and was taken as 1.0.

\subsection{Proper motions}

Pulsar proper motion is a very important quantity to be measured, so that pulsar velociaties can be determined. Pulsar timing can be used to determine the proper motions of millisecond pulsars because of their great timing stability (e.g. Nice \& Taylor 1995). However, for most pulsars, the proper motions can only be measured by determining the pulsar position precisely at two or more well-separated epochs using interferometry (e.g. Fomalont et al. 1997).

We compared the pulsar positions given in the pulsar catalog with those from the NVSS whose epoch is simply taken as MJD $=49718$, and calculated pulsar proper motions if possible. The results are listed in Table 4. Pulsars with uncertainties of proper motion larger than

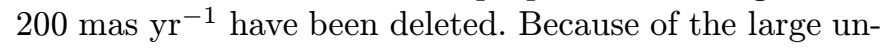
certainty of the NVSS positions, we obtained only a few significant measurements: proper motion in declination direction of PSR B1133+16, and that in right ascension of PSRs B0823+26 and B2016+28. While the former two are consistent with the previous measurements made by 
Table 4. Pulsar proper motions

\begin{tabular}{|c|c|c|c|c|c|}
\hline$\overline{\text { PSR B }}$ & $\begin{array}{l}\Delta \mathrm{RA} \\
\operatorname{arcsec}\end{array}$ & $\begin{array}{l}\Delta \text { Dec } \\
\operatorname{arcsec}\end{array}$ & $\begin{array}{l}\text { Epoch } \\
\text { MJD }\end{array}$ & $\begin{array}{c}\mu_{\alpha} \cos \delta \\
{\operatorname{mas~} \mathrm{yr}^{-1}}^{-1}\end{array}$ & 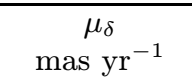 \\
\hline $0031-07$ & $-2.53 \pm 1.84$ & $2.6 \pm 1.9$ & 40690 & $-102 \pm 74$ & $-105 \pm 78$ \\
\hline $0329+54$ & $-0.61 \pm 0.44$ & $0.9 \pm 0.6$ & 40105 & $-23 \pm 16$ & $34 \pm$ \\
\hline $0450-18$ & $-1.15 \pm 1.01$ & $-2.3 \pm 1.0$ & 46573 & $-133 \pm 117$ & $267 \pm 116$ \\
\hline $0628-28$ & $1.07 \pm 1.21$ & $-1.8 \pm 1.2$ & 40585 & $42 \pm 48$ & $71 \pm$ \\
\hline $0740-28$ & $-0.53 \pm 0.80$ & $-1.3 \pm 0.8$ & 46573 & $-61 \pm 92$ & $150 \pm 93$ \\
\hline $0823+26$ & $1.21 \pm 0.94$ & $-3.7 \pm 1.0$ & 40264 & $46 \pm$ & $-142 \pm$ \\
\hline $0950+08$ & $-0.15 \pm 0.45$ & $1.3 \pm 0.6$ & 46058 & $-14 \pm 44$ & $129 \pm$ \\
\hline $1133+16$ & $-0.29 \pm 0.72$ & $6.2 \pm 0.8$ & 42364 & $-14 \pm 35$ & $307 \pm$ \\
\hline $1237+25$ & $-0.54 \pm 0.82$ & $-1.0 \pm 0.9$ & 46460 & $-61 \pm 91$ & $-112 \pm 100$ \\
\hline $1541+09$ & $0.74 \pm 1.49$ & $-2.0 \pm 1.5$ & 42304 & $36 \pm 73$ & $-98 \pm$ \\
\hline $1749-28$ & $1.72 \pm 0.53$ & $-0.8 \pm 0.9$ & 40352 & $67 \pm 20$ & $31 \pm 33$ \\
\hline $1818-04$ & $-6.74 \pm 2.69$ & $-1.5 \pm 2.7$ & 40614 & $-270 \pm 108$ & $60 \pm 108$ \\
\hline $1857-26$ & $1.08 \pm 0.95$ & $1.7 \pm 1.1$ & 46573 & $125 \pm 110$ & $-197 \pm 132$ \\
\hline $1933+16$ & $-0.14 \pm 0.58$ & $-0.9 \pm 0.7$ & 40213 & $-5 \pm 22$ & $-34 \pm$ \\
\hline $1946+35$ & $-1.34 \pm 2.56$ & $-3.1 \pm 2.9$ & 42221 & $-65 \pm 124$ & $-151 \pm 141$ \\
\hline $2016+28$ & $3 . y 42 \pm 0.79$ & $0.4 \pm 0.8$ & 40105 & $130 \pm 30$ & $15 \pm$ \\
\hline $2021+51$ & $-0.46 \pm 0.93$ & $-0.6 \pm 1.0$ & 40614 & $-18 \pm 37$ & $-24 \pm$ \\
\hline $2310+42$ & $-2.75 \pm 1.98$ & $0.4 \pm 1.4$ & 43891 & $-172 \pm 124$ & $25 \pm$ \\
\hline
\end{tabular}

Lyne et al. (1982), the latter one is marginally not. Crosschecking with Table 2 of Taylor et al. (1993), we found that all other measurements in Table 4 are consistent with (though poorer than) those given in the pulsar catalog, except for one new upper limit of PSR B0031-07. VLA A-array observations of these pulsars in Table 1 should provide much more accurate positions, and hence could produce the first measurement of the proper motions of about 20 pulsars.

PSR B0031-07 is a nearby pulsar with distance 0.68 kpc. Its proper motion upper limit indicates that the pulsar has a velocity of $470 \pm 346 \mathrm{~km} \mathrm{~s}^{-1}$, quite normal according to the pulsar velocity distribution (Lyne \& Lorimar 1994).

\section{Summary}

We identified about 97 strong pulsars from the NVSS catalog and presented the flux densities at $1.4 \mathrm{GHz}$. The parameters of linear polarization are independent, but slightly different (see Eqs. (1), and (2) above), measurements from those obtained from normal pulsar observations. Interstellar scintillation both helps and hinders the detection of pulsars. Table 1 presents all known pulsars detected by the NVSS. Well-calibrated VLA measurements of the average polarization angles of 5 strong pulsars can be used for $P A$ calibrations for pulsar observations. By comparing the pulsar positions from the pulsar catalog and those from the NVSS, we got a proper motion upper limit of PSR B0031-07.

Acknowledgements. We thank the anonymous referee for his constructive suggestions which helped to revise the paper significantly, and Drs. Dunc Lorimer, Elly Berkhuijsen, R. Wielebinski and Paul Arendt for their helpful comments. JLH is grateful for the hospitality of Prof. R. Wielebinski and Dr. R. Beck during his stay at the MPIfR, Bonn as an exchange scholar between the Chinese Academy of Sciences (CAS) and Max-Planck-Gesellschaft between 1997 May and 1998 August. He also thanks the National Natural Science Foundation of China and the Astronomical Committee of the CAS for continuous support.

\section{References}

Bailes M., et al., 1997, ApJ 481, 386

Bartel N., Morris D., Sieber W., Hankins T.H., 1982, ApJ 258 , 776

Condon J.J., Cotton W.D., Greisen E.W., et al., 1998, AJ 115, 1693

Cordes J.M., Chernoff D.F., 1998, ApJ 505, 315

Cordes J.M., Lazio T.J., 1991, ApJ 376, 123

Fomalont E.B., Goss W.M., Manchester R.N., Lyne A.G., 1997, MNRAS 286, 81

Gould D.M., Lyne A.G., 1998, MNRAS 301, 235

Gupta Y., Rickett B.J., Lyne A.G., 1994, MNRAS 269, 1035

Kaplan D.L., Condon J.J., Arzoumanian Z., Cordes J.M., 1998, ApJS 119, 75

Lorimer D.R., Lyne A.G., 1994, Nat 369, 127

Lorimer D.R., Yates J.A., Lyne A.G., Gould D.M., 1998, MNRAS 273, 411

Lyne A.G., Anderson B., Salter M.J., 1982, MNRAS 201, 503

Nice D.J., Taylor J.H., 1995, ApJ 441, 429

Prince T.A., Anderson S.B., Kulkarni S.R., Wolszczan A., 1990, Nat 346, 42

Taylor J.H., Manchester R.N., Lyne A.G., 1993, ApJS 88, 529

von Hoensbroech A., Xilouris K.M., 1997, A\&AS 126, 121 